\title{
Morbidity patterns associated with seasonal influenza A/H1N1in Swaziland
}

\author{
Vusie Lokotfwako*1, Nhlanhla Nhlabatsi', Phinda Khumalo, Siphiwe Shongwe², \\ Bongani Tsabedze', Njabulo Lukhele', Gugu Maphalala", Nomcebo Phungwayo', \\ Xolisiwe Dlamini ${ }^{1}$, Thulani Maphosa ${ }^{2}$ and Harriet Nuwagaba-Biribonwoha ${ }^{2}$
}

'Epidemiology and Disease Control Unit, Ministry of Health, Mbabane, Swaziland; ${ }^{2}$ ICAP Columbia University, Mbabane, Swaziland

\section{Objective}

To establish morbidity patterns of influenza A/H1N1 in Swaziland from $10^{\text {th }}$ July to $15^{\text {th }}$ August 2017.

\section{Introduction}

Influenza infection is caused by the influenza virus, a singlestranded RNA virus belonging to the Orthomyxoviridae family. Influenza viruses are classified as types A, B and C. Influenza A and $\mathrm{B}$ viruses can cause epidemic disease in humans and type $\mathrm{C}$ viruses usually cause a mild, cold-like illness. The influenza virus spreads rapidly around the world in seasonal epidemics, resulting in significant morbidity and mortality. On the $10^{\text {th }}$ of July 2017 , a case of confirmed Influenza A/H1N1 was reported through the immediate disease notification system from a private hospital in the Hhohho region. A 49 year old female was diagnosed of Influenza A/H1N1 after presenting with flu-like symptoms. Contacts of the index case were followed and further positive cases were identified.

\section{Methods}

Upon identification of the index case, the rapid response teams conducted further investigations. Two nasal swaps from each sample were taken and sent to a private laboratory in South Africa for the detection of the virus RNA using RT-PCR to assess for the presence Influenza A, B and Influenza A/H1N1. Further laboratory results were sourced from a private laboratory to monitor trends of influenza. Data was captured and analyzed in STATA version 12 from STATA cooperation. Descriptive statistics were carried out using means and standard deviations. The Pearson Chi square test and student $t$ test were used to test for any possible association between influenza A/ H1N1 and the explanatory variables (age and sex).

\section{Results}

Surveillance data captured between $10^{\text {th }}$ July 2017 and $15^{\text {th }}$ August 2017 indicated that a total of 87 patients had their samples taken for laboratory confirmation. There were 45 females and 42 males and the mean age was 27 years $(S D=17)$. At least 25 of the 87 patients tested positive for influenza A while only 1 tested positive for influenza $\mathrm{B}$. The prevalence of influenza $\mathrm{A} / \mathrm{H} 1 \mathrm{~N} 1$ was $16 \%$. The prevalence of influenza $\mathrm{A} / \mathrm{H} 1 \mathrm{~N} 1$ among males was $19 \%$ compared to $13 \%$ in females; however the difference was not statistically significant $(p=0.469)$. There was no association noted between age and influenza $\mathrm{A} / \mathrm{H} 1 \mathrm{~N} 1$ ( $\mathrm{p}=0.427)$. Upon further sub-typing results indicated that the circulating strain was influenza A/H1N1 pdm 09 strain which is a seasonal influenza. The epidemic task forces held weekly and ad-hoc meetings to provide feedback to principals and health messaging to the general population to allay anxiety.

\section{Conclusions}

Though WHO has classified the influenza A/H1N1 strain pdm 2009 as a seasonal influenza, surveillance remains important for early detection and management. There is therefore an urgent need to set up sentinel sites to monitor and understand the circulating influenza strains. Health promotion remains crucial to dispel anxiety as the general public still link any influenza to the 2009 pandemic influenza. Finally the Ministry of Health should consider introducing influenza vaccines into the routine immunization schedule especially for children.

Keywords

Morbidity; Influenza; Swaziland

\section{Acknowledgments}

1. Ministry of Health, Mbabane, Swaziland

2. ICAP, Columbia University, Mailman School of Public Health, Mbabane, Swaziland

3. Columbia University, Department of Epidemiology, Mailman School of Public Health, New York, United States of America

\section{References}

1. Global Epidemiological Surveillance Standards for Influenza. 2014 [cited 201515 April]; Available from: http://www.who.int/influenza/ resources/documents/influenza_surveillance manual/en/.

2. Human cases of influenza at the human-animal interface, 2013. Wkly Epidemiol Rec, 2014. 89(28): p. 309-20.

3. WHO Global Influenza Surveillance Network. Manual for the laboratory diagnosis and virological surveillance of influenza. 2011 [cited 2015 April27]; Available from: http://www.who.int/influenza/ gisrs_laboratory/manual_diagnosis_surveillance_influenza/en/.

\section{*Vusie Lokotfwako \\ E-mail:vusielokza@gmail.com}

